\title{
A biophysical mechanism may control the collinearity of Hoxd genes during the early phase of limb development
}

Spyros Papageorgiou*

Institute of Biology, NRC 'Demokritos', Aghia Paraskevi Attikis, Athens, Greece

*Correspondence to: Tel: +30 210 8954920; Fax: +30 210 8955008; E-mail: spapage@bio.demokritos.gr

Date received (in revised form): 17th November, 2008

\begin{abstract}
A biophysical model has been proposed which deals with the observed collinearity of Hox gene expressions in developing vertebrate limbs. It is assumed that physical forces gradually dislocate the genes of the Hoxd cluster from inside the chromosome territory into the interchromosome domain, where the genes are activated.

In particular, the action of Coulomb electric forces has been estimated in detail. Genetic engineering experiments (deletions, duplications and transpositions) were recently reported for Hoxd expression during limb development. Here, we analyse these results and show that the biophysical model explains them successfully.
\end{abstract}

Keywords: Hox collinearity, gene deletion, duplication, transposition

\section{Introduction}

The collinearity of Hox gene expressions is a surprising feature observed during animal development along the primary and secondary body axes. ${ }^{1,2}$ These gene expressions follow in space and time the sequence of gene ordering from $3^{\prime}$ to $5^{\prime}$ in the $H o x$ cluster. $^{2,3}$ In the Hoxd cluster, the genes are lined up in the $3^{\prime}$ to $5^{\prime}$ direction and their expression domains (Hoxd1, Hoxd2, Hoxd3,...) along the anteroposterior axis follow a peculiar pattern: the anterior border of expression for each gene is shifted in the anterior-posterior direction - for example, the anterior border of Hoxd1 expression is more anterior than the anterior border of Hoxd2 expression etc. Thus, a pattern of partially overlapping expression domains along the head to tail axis is formed ('spatial collinearity'). ${ }^{2}$ Furthermore, the genes of the Hoxd cluster are activated one after the other in the same $3^{\prime}$ to $5^{\prime}$ order ('temporal collinearity').,3 Tarchini and Duboule ${ }^{4}$ have put forward a model in which, during early limb budding (first wave), two regulatory influences control the activation of Hoxd genes: one mechanism is located at the telomeric $\left(3^{\prime}\right)$ side of the cluster (early limb control regulation; ELCR) and the other relies on sequences centromeric to the cluster (POST). Thus, in order to describe the data in terms of biomolecular processes only, two opposing collinearity mechanisms have to be introduced. ${ }^{4}$

\section{The biophysical model - Comparison with experiments}

An alternative biophysical model was introduced, dealing with the initial stages of limb development. ${ }^{5}$ According to this model, the inactive Hox cluster is anchored inside the chromosome territory (CT), where the gene regulatory regions are inaccessible for transcription. Physical forces may pull the Hox fibre towards the interchromosome domain (ICD). Just outside the CT, the genes co-localise both in cis and in trans with local concentrations of transcription factories, and gene 
transcription is initiated (Figure 1). The intensity of a Hox gene transcription decreases when the gene moves in the ICD away from the CT boundary. Since the nature of the physical forces is unknown, we consider the electric Coulomb forces as plausible candidates. ${ }^{5}$ Normally, the Hox cluster is negatively charged, and positive polar molecules are deposited at some distance from the cluster, as shown schematically in Figure 1. The positive charges, $P$, in every cell
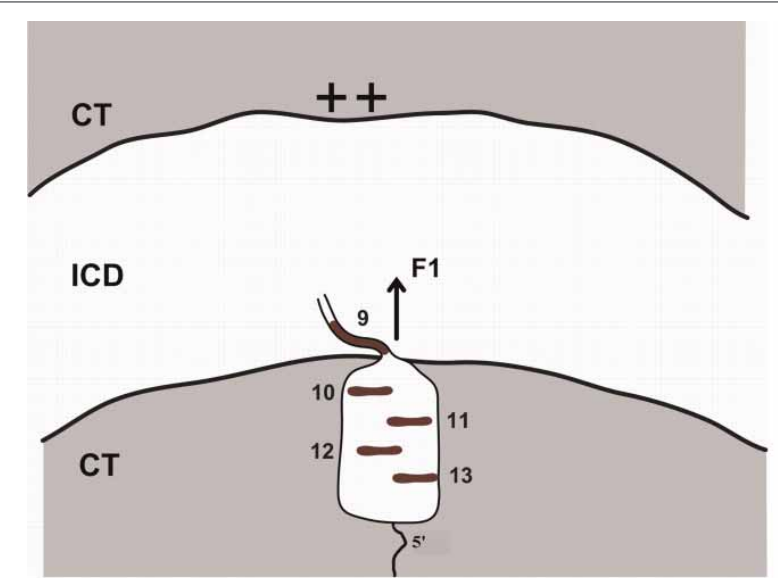

(a)

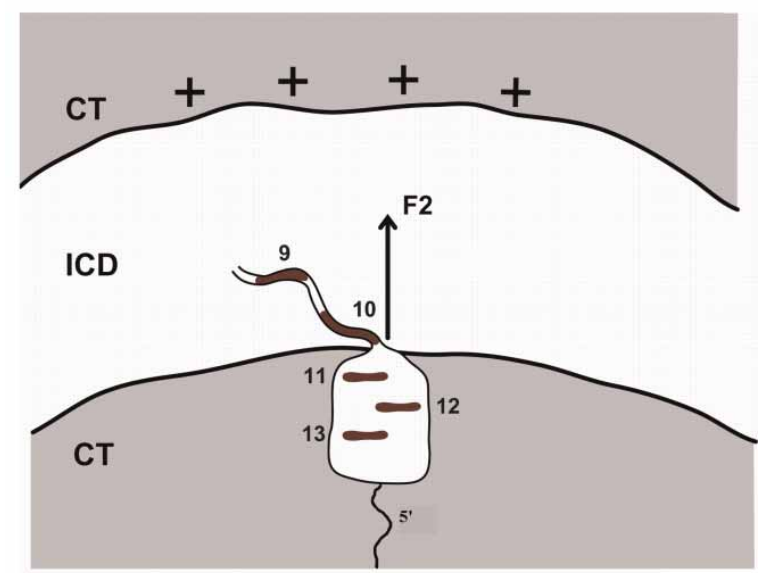

(b)

Figure I. Hoxd extrusions. When no positive charges are deposited opposite the $5^{\prime}$ Hoxd subcluster, the subcluster remains inside the chromosome territory (CT). (a) For a low distribution of positive charges $P(++)$, a small Coulomb force $F I$ is created and one gene (Hoxd9) is extruded in the interchromosome domain (ICD). This occurs in domain D9 for cells in which the morphogen concentration is above the corresponding threshold T9. (b) For increased positive charges $P(++++)$, the force $F 2$ is stronger and two consecutive genes (Hoxd9 and HoxdlO) are extruded. This occurs in domain DI0, where the morphogen is above threshold TIO. nucleus correspond to an extracellular morphogen, which forms a gradient along the anteroposterior axis; ${ }^{5} P$ is high in the posterior cells and decreases gradually towards the anterior cells. A Coulomb force is created that pulls the Hox cluster; it decondenses the gene fibre and translocates the Hox genes in the ICD, following the $3^{\prime}$ to $5^{\prime}$ order (Figure 1).

The biophysical model does not merely describe Hox gene collinearity. Its aim is to explain it and to provide the mechanism that causes the modes of Hox gene expression by integrating and coordinating actions at both the multicellular and subcellular levels. Of course, the control regions, such as ELCR and any other regulatory elements that have yet to be discovered, are essential tools of the biochemical machinery that participate in the realisation of Hox gene expression. We believe, however, that this essential biomolecular pathway is triggered and controlled by a more fundamental, single and unidirectional mechanism, such as the one presented here. The biophysical model reproduces spatial, temporal and quantitative collinearities well. ${ }^{5}$

\section{Gene deletions, duplications and transpositions}

Duboule and collaborators ${ }^{4,6,7}$ have performed detailed and extensive experiments of Hoxd gene deletions and duplications in order to explore the features of collinearity during vertebrate limb development. Their findings for the early phase of limb development ${ }^{4}$ are highly illuminating, and it is worth comparing them with the biophysical model predictions.

Suppose the subcluster of the $5^{\prime}$ Hoxd genes has an evenly distributed negative charge $N(N=10$ in arbitrary units) and that every gene region (Hoxd9, ... Hoxd13) carries a charge $N / 5=2$. Before activation of the $5^{\prime}$ Hoxd subcluster, all genes are embedded inside the CT. In this case, we can set $P=0$ and the Coulomb force is 0 . For activation of the first gene (Hoxd9), we set $P=2$ (in arbitrary units) and $P=10$ when all genes (Hoxd 9, . . , Hoxd13) are extruded in the ICD.

The measure of the Coulomb force $F$ acting on the subcluster is proportional to the positive and 
negative charges. For our rough comparative estimates, we can assume:

$$
F=P \times N
$$

In this truncated Coulomb equation, we neglect the dependence of $F$ on the distance between positive and negative charges, and the Coulomb's constant is set at 1 (in appropriate units). The Coulomb force causes gene fibre decondensation and extrusion of length $L$. We assume, in a first approximation, that this extrusion length $L$ is proportional to $F$ in the spirit of Hooke's law of elastic spring expansion. Deletion of one Hoxd gene region causes a reduction in the negative charge of the cluster; therefore, the Coulomb force will decrease and consequently the extrusion length of the Hoxd fibre will be shortened. The opposite will occur with a duplication of one Hoxd gene region in the cluster: the larger the duplication, the longer the gene fibre extrusion. In the supplementary material, we present some indicative experiments of gene deletions, duplications and transpositions 8 and compare them with the biophysical model predictions. The agreement is astonishing.

\section{Discussion}

In an analysis of the Hoxd locus in mouse development, activation along the primary anteroposterior axis is accompanied by fibre extrusion from the $\mathrm{CT}^{9}$ In the limb bud, at the same stage (E 9.5), however, no looping out is detected, even though the locus is still decondensed. A further confirmation of this difference is needed, taking into account that at that stage Hoxd expressions (eg Hoxd4) in the mouse limb bud are much weaker than in the tailbud. ${ }^{9}$

The biophysical model explains how collinearity phenomena occur during the early stages of limb development. At later stages, when all Hoxd genes are expressed, secondary regulations and gene interactions modulate their expressions and the complex landscape of digits starts emerging. Recently, Montavon et al. ${ }^{7}$ analysed experimentally and theoretically the features of quantitative collinearity in developing digits. They used some of their essential findings to formulate a model for the differential importance of gene rank - the relative position of Hoxd genes with respect to the $5^{\prime}$ extremity of the cluster. For instance, they observed that when Hoxd13 (the most strongly expressed gene) was deleted, the gene that substituted for it in the last $5^{\prime}$ position was expressed with an increased intensity. It is instructive to view this result in the framework of the biophysical model: as soon as Hoxd13 is activated, the whole gene fibre is extruded and Hoxd13 is located closest to the surface of the CT, and its expression is therefore strongest. (This occurs in the posterior domain of the limb bud.) When Hoxd13 is deleted, the extruding Coulomb force $F$ decreases and the extruded fibre retreats toward the interior of the CT. The consecutive gene (eg Hoxd12) is dislocated towards the CT surface and consequently its expression is strengthened. This is the essence of the observation by Montavon et al. ${ }^{7}$ and it indicates, on the one hand, that this observation constitutes additional evidence in favour of the biophysical model. On the other hand, it shows that our interpretation of quantitative collinearity can be extended to all stages of limb development. Finally, the above authors have managed to establish a quasi-linear relationship between the total transcriptional activity at the locus and the number of transcription units - e.g. active genes in developing digits (Figure $4 \mathrm{~b}$ in reference 7 ). This remarkable correlation supports our hypothesis of Coulomb force variations and the corresponding proportional changes in the extruded fibre lengths (reminiscent of Hooke's linear law of elastic expansions).

\section{References}

1. Lewis, E.B. (1978), 'A gene complex controlling segmentation in Drosophila', Nature Vol. 276, pp. 565-570.

2. Nolte, C. and Krumlauf, R. (2007), 'Expression of Hox genes in the nervous system of vertebrates', in: Papageorgiou, S. (ed.), 'HOX Gene Expression', Landes Bioscience and Springer Science+Business Media, New York, NY, USA.

3. Nelson, C.E., Morgan, B.A., Burke, A.C. et al. (1996), 'Analysis of Hox gene expression in the chick limb bud', Development Vol. 122, pp. 1449-1466. 
4. Tarchini, B. and Duboule, D. (2006), 'Control of Hoxd genes' collinearity during early limb development', Developmental Cell Vol. 10, pp. 93-103.

5. Papageorgiou, S. (2006), 'Pulling forces acting on Hox gene clusters cause expression collinearity', Int. J. Dev. Biol. Vol. 50, pp. 301-308.

6. Kmita, M., Fraudeau, N., Herault, Y. and Duboule, D. (2002), 'Serial deletions and duplications suggest a mechanism for the collinearity of Hoxd genes in limbs', Nature Vol. 420, pp. 145-150.

7. Montavon, T., Le Garrec, J.-F., Kerszberg, M. and Duboule, D. (2008), 'Modeling Hox gene regulation in digits reverse collinearity and the molecular origin of thumbness', Genes Dev. Vol. 22, pp. 346-359.

8. Morey, C., Da Silva, N.R., Kmita, M. et al. (2008), 'Ectopic nuclear reorganization driven by a Hoxb1 transgene transposed into Hoxd', J. Cell Sci. Vol. 121, pp. 571-577.

9. Morey, C., Da Silva, N.R., Perry, P. and Bickmore, W.A. (2007), 'Nuclear reorganization and chromatin decondensation are conserved, but distinct, mechanisms linked to Hox gene activation', Development Vol. 134, pp. 909-919.

\section{Supplementary material}

We can establish the following correlations between cell locations, Coulomb forces and subsequent Hoxd gene extrusions and subsequent activations.

1) For cells expressing only one gene (Hoxd 9):

$$
P=2, \quad N=10, \quad F=2 \times 10=20, \quad L=1
$$

(Figure 1a), Domain D9

2) For cells expressing two genes (Hoxd9 and Hoxd10):

$$
P=4, \quad N=10, \quad F=4 \times 10=40, \quad L=2
$$

(Figure 1b), Domain D10 < Domain D9

3) For cells expressing three genes (Hoxd9, Hoxd10 and Hoxd11):

$P=6, \quad N=10, \quad F=6 \times 10=60, \quad L=3$, Domain D11 < Domain D10 < Domain D9... etc.

In experiments where one gene of the subcluster is deleted, the negative charge of the cluster will decrease to $N=8$ and the length of the extruded fibre will be shorter. Two gene deletions will produce an even bigger reduction in the length of the extruded fibre. By contrast, when one gene is duplicated, $N$ will increase to 12 and the extruded fibre will be longer etc.
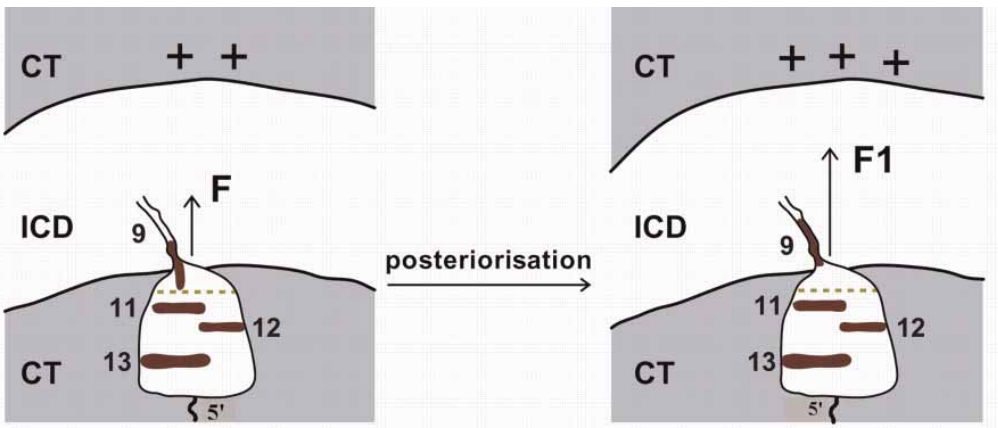

(a)
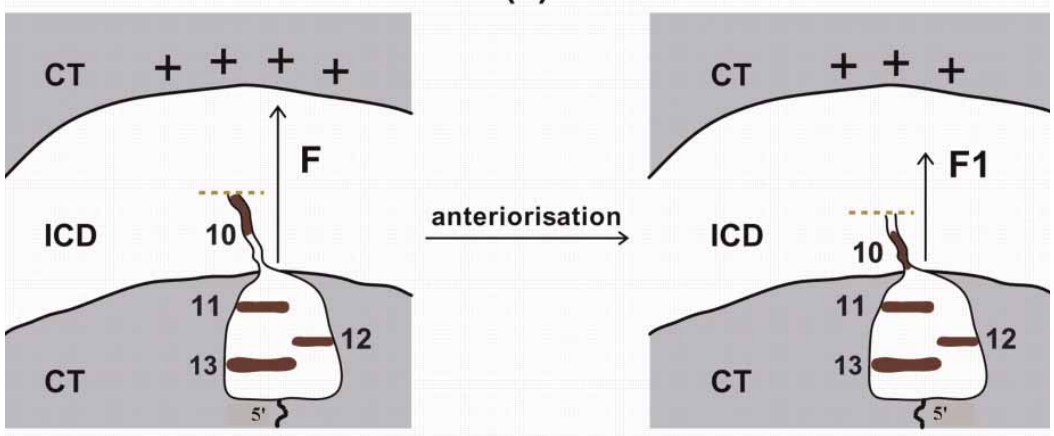

(b)

Figure IS. Hoxd gene deletions. (a) Posterior gene deletions. Consider activation of Hoxd 9 when Hoxd 10 is deleted (left). $N$ decreases and the force $F$ is smaller than $\mathrm{FI}$; therefore, Hoxd 9 retreats inside the CT. For the extrusion of Hoxd9, $P$ has to increase to more posterior locations (right). (b) Anterior gene deletions. Consider activated gene HoxdI0. When anterior gene Hoxd9 is deleted, $F$ decreases (left) but is still higher than the force $F I$ needed for the extrusion of the first gene (Hoxd9), hence, Hoxd I 0 expression shifts to more anterior cells with lower $P$ (right). 
In the following, we estimate how deletions and duplications modify gene expressions, and particularly in domains D9, D10 and D11.

\section{(a) Posterior gene deletions}

Consider cells in which Hoxd9 is normally activated (domain D9). The normal force acting on the fibre is (at least): $F 1=2 \times 10=20$ (Figure 1a). If posterior gene Hoxd10 is deleted, the negative charge $N$ of the cluster decreases to 8 and the pulling force on the fibre becomes $F=2 \times 8=$ 16, which is below the normal value of 20 . Consequently, Hoxd9 will retreat towards the interior of the CT (Figure 1Sa; left).

For the complete extrusion of Hoxd 9, a stronger force is needed and this is achieved by a posterior shift to cells where the morphogen value is higher, as is the corresponding $P$ value: $P(++\rightarrow+++)$.
If more than one posterior gene is deleted, the posterior shift of Hoxd9 activation will increase. Tarchini and Duboule ${ }^{4}$ designated this as an unexpected observation. For the biophysical model, this posteriorisation is an obvious outcome.

\section{(b) Anterior gene deletions}

This case is more involved because, besides the charge modification of the cluster, the intervention also affects the length of the extruded gene fibre (Figure 1Sb). Consider, for example, cells in which Hoxd 9 and Hoxd10 are activated, where the normal extrusion force is (at least) $F 2=40$. If Hoxd 9 is deleted, the extrusion force on the fibre is reduced to $F=4 \times 8=32$, which is lower than 40. In this situation, Hoxd10 is the first extruded gene. For this first gene, we estimated that the necessary extrusion force is $F 1=20$ at least.
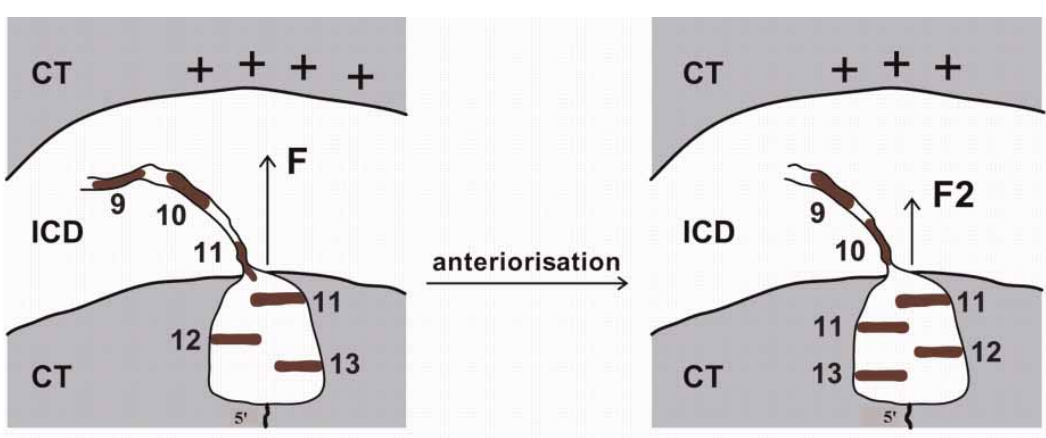

(a)
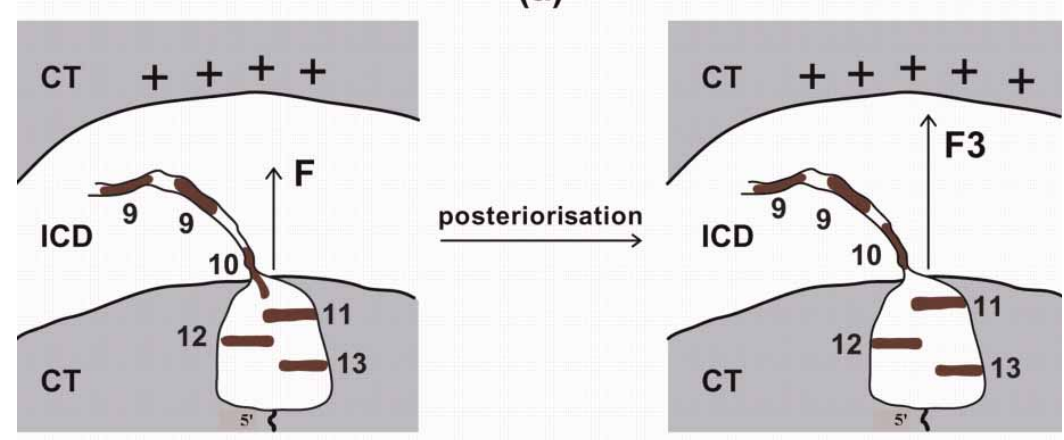

(b)

Figure 2S. Hoxd gene duplications. Consider cells expressing Hoxd9 and Hoxd IO. (a) Posterior gene duplications. If Hoxd I is duplicated (left), $F$ increases above the minimum value F2; therefore, Hoxd 10 can be expressed ectopically in more anterior locations (right). (b) Anterior gene duplication. If Hoxd9 is duplicated, the pulling force increases (left) but the increase is not sufficient to reach F3 for the extrusion of three genes; therefore, $P$ must increase for activation of the third gene (Hoxd I 0 ), whose expression is then shifted posteriorly (right). 
For activation of Hoxd10, F can be reduced to F1, and this occurs at more anterior cell positions with a lower $P$ value: $\mathrm{P}(++++\rightarrow+++)$. An ectopic expression of Hoxd10 in the anterior region of the limb bud will be observed, associated with a premature expression of the gene. This was verified by Tarchini and Duboule. ${ }^{4}$

\section{(c) Posterior gene duplications}

Compared to gene deletions, gene duplications have an opposite effect on Hoxd gene expressions. Consider, again, cells in which Hoxd 9 and Hoxd10 are activated, where the pulling force is (at least) $F 2=40$. If posterior gene Hoxd11 is duplicated (Figure 2Sa), the charge of the cluster will increase to $N=12$.

The pulling force on the fibre will become: $F=$ $4 \times 12=48$, which is higher than F2. Activation of Hoxd10, however, can start with F2; therefore, F can be reduced to this value. This is achieved by an anterior shift of D10; in this case, the morphogen decreases, and so does $P(++++\rightarrow+++)$ and $F \rightarrow F 2$. The result is an anteriorisation and premature expression of Hoxd10.

Let us examine Hoxd10 expression at a position and time without Hoxd11 duplication. At this position, after Hoxd11 duplication, Hoxd10 will be abnormally translocated further inside the ICD, away from the CT border. A downregulation of Hoxd10 expression is expected at this position (quantitative collinearity). This was verified by Kmita et al. ${ }^{6}$

\section{(d) Anterior gene duplications}

Consider the case of two activated genes (Hoxd9 and Hoxd10), where $F 2=40$. If anterior gene Hoxd 9 is duplicated, the pulling force on the fibre increases from $F 2$ to $F=48$. For activation of Hoxd10, however, this increase is not sufficient, since now three genes must be extruded. The corresponding pulling force for three genes is $F 3=$ 60 (Figure 2Sb). For a stronger force F3, P must take a higher value: $P(++++\rightarrow+++++)$. Therefore, after an anterior gene duplication, the expression of Hoxd10 will be shifted posteriorly, in agreement with the findings of Tarchini and Duboule. $^{4}$

These calculations can be applied to extended gene deletions and duplications incorporating several genes from the entire Hoxd cluster. Following the above rules, the estimation of anteriorisations or posteriorisations is straightforward, and the results agree with the findings of Duboule and co-workers. The conclusion is that Hoxd gene deletions and duplications strongly support the present electric force mechanism of the biophysical model.

\section{Hoxb1 transposition in Hoxd}

Recently, the Hoxb1 transgene was transposed at the $5^{\prime}$ end of the Hoxd cluster. ${ }^{8}$ The results from this transposition are in agreement with the biophysical model. For example, in the wild-type mouse embryo at stage E7.5, Hoxd13 is not expressed in the primitive streak tissue. By contrast, Hoxd13 is activated when the Hoxb1-Lac Z reporter is inserted at the $5^{\prime}$ end of the Hoxd cluster. This finding is explained by the biophysical model, since, in analogy with the Hoxd regions, the Hoxb1 transgene carries a negative charge and the total $N$ increases in the transgene embryo (as analysed in detail above). The pulling force on the gene fibre therefore increases, so that Hoxd13 loops out of the $\mathrm{CT}$ and its activation is possible. In the wild-type limb bud at E9.5, the Hoxd region, although decondensed, does not extrude out of the $\mathrm{CT}^{8}$. In the Hoxb1-LacZ embryo, however, Hoxd does loop out and this can be attributed, again, to the increased force of attraction that pulls the gene fibre out of the CT. 